\title{
Symmetric Four-mass Schubart-like Systems
}

\author{
Winston L. Sweatman \\ Institute of Natural and Mathematical Sciences, \\ Massey University, Albany, Auckland, New Zealand \\ email: w.sweatman@massey.ac.nz
}

\begin{abstract}
The general four-body problem can be simplified by considering the special case where the system contains two pairs of identical masses and is symmetrical. The simple models that occur may aid our understanding of the general problem. Systems that arise from Schubartlike interplay orbits are an important feature of the dynamics.
\end{abstract}

Keywords. celestial mechanics, symmetrical four-body problem, binary-binary interactions, Caledonian four-body problem, Schubart-like interplay orbits

\section{Introduction}

Although somewhat simpler, a four-body system with symmetry may provide some insight into understanding the general four-body problem (Sweatman 2015). The Caledonian four-body problem, Fig. 1, is a four-body system which has a rotational symmetry about its centre of mass (Roy \& Steves 1998, 2001; Steves \& Roy 1998). The symmetry requires masses $m_{1}$ and $m_{3}$ to be equal and images of one another on opposite sides of the centre of mass. Likewise, masses $m_{2}$ and $m_{4}$ are equal and images. If motion is strictly one-dimensional, the Caledonian four-body problem becomes the symmetrical collinear four-body problem (Sweatman 2002, 2006; Sekiguchi \& Tanikawa 2004). For this the masses remain in the order $m_{1}, m_{2}, m_{4}, m_{3}$. The inevitable collisions between mass pairs are resolved with an elastic bounce, the limit of a planar encounter as eccentricity tends to unity. The system's state is given by the positions and momenta of $m_{1}$ and $m_{2}: x_{1}$ and $x_{2}$, and $w_{1}=2 m_{1} \dot{x}_{1}$ and $w_{2}$, respectively, with Hamiltonian

$$
H=\frac{w_{1}^{2}}{4 m_{1}}+\frac{w_{2}^{2}}{4 m_{2}}-\frac{m_{1}^{2}}{2 x_{1}}-\frac{m_{2}^{2}}{2 x_{2}}-\frac{2 m_{1} m_{2}}{x_{1}+x_{2}}-\frac{2 m_{1} m_{2}}{x_{1}-x_{2}} .
$$

We regularise equations of motion using the square-root of inter-mass distances, conjugate momenta and rescaled time (Sweatman 2002, 2006). The Caledonian four-body problem can be handled similarly with Levi-Civita regularisation (Sivasankaran et al. 2010).

As for three bodies a key dynamical feature of the one-dimensional four-body problem is the periodic orbit analogous to Schubart's orbit (Schubart 1956). In this orbit, there are alternately interactions between masses $m_{2}$ and $m_{4}$ at the centre, and simultaneous interactions, between $m_{1}$ and $m_{2}$ and between $m_{4}$ and $m_{3}$, on the outside.

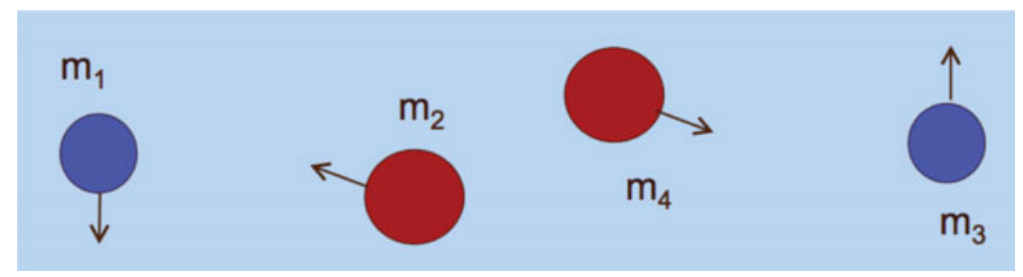

Figure 1. The Caledonian four-body problem. 


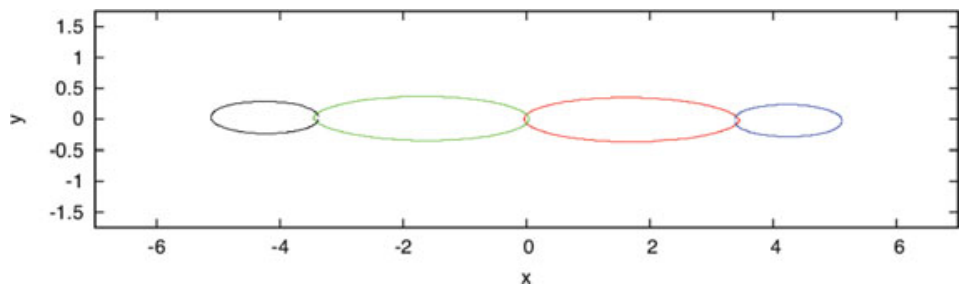

Figure 2. A planar orbit near the equal mass Schubart-like orbit. Approximate initial positions are $\pm 3.45413106, \pm 3.38409367$, with transverse velocities $\mp 3.79430129, \pm 3.70734831$.

\section{Families of Schubart-like Interplay Orbits}

The collinear equal-mass Schubart-like orbit is found by progressive refinement of a Poincaré section (Sweatman 2002). It is collinearly stable but spatially unstable.

From the equal-mass Schubart-like orbit, families of related periodic orbits can be found using approaches similar to those of three-body investigations (Schubart 1956; Hénon 1976, 1977; Mikkola \& Hietarinta 1991; Hietarinta \& Mikkola 1993). In one-dimension, a family of Schubart-like interplay orbits arises from different mass ratios (Sweatman 2006). Some of these orbits are spatially stable. The orbits are found by applying differential correction. Beginning with the known equal-mass solution, the results for previous mass ratios are used to generate approximate initial conditions for the current mass ratio. The orbit is integrated through one period and the difference between initial and final points is used to produce improved initial conditions. The beginning and end of the period were taken to be times at which the inter-body distances were at a fixed ratio.

Hénon (1976) generated a family of periodic orbits in the plane from Schubart's orbit. Ongoing investigation suggests a similar family of planar orbits for the corresponding four-body case. Fig. 2 shows one such orbit which is relatively close to the equal-mass Schubart-like interplay orbit. Each outer mass orbits the closer inner mass. The inner masses alternately pass around one another, and the corresponding outer mass. Again, differential correction is used to find this periodic orbit. The search started with collinear masses close to the positions of simultaneous double collision for the Schubart-like orbit. Velocities perpendicular to the line of the bodies are determined to provide a small fixed non-zero angular momentum. The beginning and end of the period are taken to be maxima of the distance from $m_{2}$ to $m_{4}$. The caption has approximate initial conditions.

\section{References}

Hénon, M. 1976, Cel. Mech. Dyn. Astron., 13, 267

Hénon, M. 1977, Cel. Mech. Dyn. Astron., 15, 243

Hietarinta, J. \& Mikkola, S. 1993, Chaos, 3,183

Mikkola, S. \& Hietarinta, J. 1991, Cel. Mech. Dyn. Astron., 51, 379

Roy, A. E. \& Steves, B. A. 1998, Planet. Space Sci., 46, 1475

Roy, A. E. \& Steves, B. A. 2001, Cel. Mech. Dyn. Astron., 78, 299

Schubart, J. 1956, Astron. Nachr., 283, 17

Sekiguchi, M. \& Tanikawa, K. 2004, PASJ, 56, 235

Sivasankaran, A., Steves, B. A., \& Sweatman, W. L. 2010, Cel. Mech. Dyn. Astron., 107, 157

Steves, B. A. \& Roy, A. E. 1998, Planet. Space Sci., 46, 1465

Sweatman, W. L. 2002, Cel. Mech. Dyn. Astron., 82, 179

Sweatman, W. L. 2006, Cel. Mech. Dyn. Astron., 94, 37

Sweatman, W. L. 2015, Symmetric four-body problems, in: M. Cojocaru, I. S. Kotsireas, R. N. Makarov, R. Melnik \& H. Shodiev (eds.), Interdisciplinary Topics in Applied Mathematics, Modeling, and Computational Science, Springer Proceedings in Mathematics and Statistics, Volume 117, in press 